\title{
Length of hospital stay after hip fracture and risk of early mortality after discharge in New York state: retrospective cohort study
}

\begin{abstract}
Study question Can the length of hospital stay for hip fracture affect a patient's risk of death 30 days after discharge?

Methods In a retrospective cohort study, population based registry data from the New York Statewide Planning and Research Cooperative System (SPARCS) were used to investigate 188208 patients admitted to hospital for hip fracture in New York state from 2000 to 2011. Patients were aged 50 years and older, and received surgical or non-surgical treatment. The main outcome measure was the mortality rate at 30 days after hospital discharge.

Study answer and limitations Hospital stays of 11-14 days for hip fracture were associated with a $32 \%$ increased odds of death 30 days after discharge, compared with stays lasting one to five days (odds ratio 1.32 (95\% confidence interval 1.19 to 1.47$))$. These odds increased to $103 \%$ for stays longer than 14 days (2.03 (1.84 to 2.24$)$ ). Other risk factors associated with early mortality included discharge to a hospice facility, older age, metastatic disease, and non-surgical management. The 30 day mortality rate after discharge was $4.5 \%$ for surgically treated patients and $10.7 \%$ for non-surgically treated patients. These findings might not be generalizable to populations in other US states or in other countries. The administrative claims data used could have been incomplete or include inaccurate coding of diagnoses and comorbid conditions. The database also did not include patient socioeconomic status, which could affect access to care to a greater extent in New York state than in European countries. Specific cause of death was not available because few autopsies are performed in this population.

What this study adds By contrast with recent findings in Sweden, decreased length of hospital stay for hip fracture was associated with reduced rates of early mortality in a US cohort in New York state. This could reflect critical system differences in the treatment of hip fractures between Europe and the USA.
\end{abstract}

Funding, competing interests, data sharing University of Rochester grant from the Clinical Translational Science Institute for statistical analyses used in this work (National Institutes of Health (UL1 TR000042)) and the National Institutes of Health (K-08 AR060164-01A). No competing interests declared. Data may be obtained through SPARCS at https://www.health.ny.gov/statistics/sparcs/access/.

\section{Introduction}

Hip fracture is common, costly, and often associated with poor outcomes among older adults. Mortality rates after hip fracture have changed little over the past 30 years despite surgical and medical improvements. ${ }^{1}$ Nonetheless, mortality rates are universally measured in registries and large databases. Length of hospital stay has fallen over time in both Europe and the United States. ${ }^{2}$ Recent work has suggested that a longer hospital stay after hip fracture is associated with decreased mortality following discharge. ${ }^{3}$

Healthcare systems in Europe and the USA differ in both length of hospital stay and usual discharge destination. In the USA, $90 \%$ of patients with hip fracture are discharged to rehabilitation facilities and receive much of their follow-up care in these facilities. ${ }^{4}$ Rehabilitation facilities in the USA serve a transitional role and could help prevent early mortality after discharge. ${ }^{5}$ By contrast, more patients are discharged home from hospital after hip fracture in other countries such as the United Kingdom. ${ }^{6}$

The New York Statewide Planning and Research Cooperative System (SPARCS) database offers a unique opportunity to measure postsurgical mortality in a complete dataset of US patients and has been used previously to study hip fractures.? We hypothesized that SPARCS data would demonstrate that a shorter hospital stay is associated with lower rates of mortality at 30 days after hip fracture. 


\section{Methods \\ Patients}

This study used the SPARCS database to identify 188938 patients aged 50 years or older admitted to a public or private hospital in New York state between 1 January 2000 and 31 December 2011 with a hip fracture. SPARCS is a comprehensive, all payer administrative database that collects patient level data from all non-federal, acute care facilities in New York state (233 hospitals during the study period). The database collects information including patient demographics, diagnoses, procedures, and charges for every inpatient hospital admission, ambulatory surgical procedure, and emergency department admission. Individuals are assigned a unique, encrypted identification code, allowing for longitudinal analyses. Estimated reporting completeness obtained from SPARCS inpatient annual reports during the study period (2000-11) ranged from $95 \%$ to $100 \%$, with an average of more than $98 \%$.

We identified patients in hospital discharge records originating from non-rehabilitation hospitals in New York state and containing an admitting or primary diagnosis code for a hip fracture using the ICD-9-CM classification (international classification of diseases, 9th revision, clinical modification; codes 820.00-820.03, 820.09, 820.20-820.22, 820.8). Patients were excluded if they did not have the unique personal identifier (encrypted combination of name, date of birth, social security number, hospital, and date of admission; $n=68$ ) or there were duplicate unique identifiers $(\mathrm{n}=662)$. The remaining 188208 patients were included in the analysis. We determined 30 day mortality data using a linkage with the New York State Department of Vital Statistics and New York City Department of Vital Statistics. Patients who died during the hospital stay were excluded from analyses.

We identified surgical procedures and dates with ICD-9 procedure codes $(81.51,81.52,78.55,73.35,79.15,79.25$, or $79.05)$ and associated procedure dates. Time to surgery was defined as days between admission and procedure date. We defined comorbidities on the basis of the Charlson comorbidity index using a modified Stata interpretation of a SAS software program obtained from the Boston College Department of Economics in its series statistical software components. ${ }^{8}$ Table $1 \Downarrow$ shows the coding algorithm used to define comorbidities.

\section{Statistical methods}

The primary analysis was designed to determine whether length of hospital stay (categorically defined as 1-5 days, 6-10 days, 11-14 days, and $>14$ days) was associated with mortality rates at 30 days after hospital discharge. We assessed differences between categorical groups using $\chi^{2}$ analysis and differences between continuous variables using Student's $t$ test. Patients who died before hospital discharge were excluded from analyses related to post-discharge mortality. We made survival estimates using the Kaplan-Meier method. We determined risk of mortality using Cox proportional hazards models while adjusting for comorbid conditions. However, the proportional hazards assumption was violated on testing with $\mathrm{P}<0.05$; therefore, multivariate logistic regression analysis evaluated risk of mortality after hospital discharge on the basis of categorical length of stay, while adjusting for covariates in the univariate model.

To test whether the association between length of stay and odds of death within 30 days after discharge was a time dependent interaction, we created a product interaction term. The likelihood ratio test found the test statistic was equal to 15.4492 with three degrees of freedom and a P value of 0.00147 . Therefore, the product interaction term was included in the primary multivariate logistic regression model as a covariate.

To evaluate whether non-surgically treated patients were dying soon after hospital discharge and contributing to increased mortality at 30 days, we created three models. The models included all patients with a hip fracture regardless of surgical management, where non-surgical management was included as a covariate; patients treated surgically only; and patients treated non-surgically only.

\section{Subgroup and sensitivity analyses}

We did several additional analyses to control for confounding factors. To control for a reduction in length of hospital stay over the 12 year study period, the relation between length of stay and 30 day post-discharge mortality was evaluated for each year separately in both adjusted and unadjusted logistic regression models. To evaluate whether risk of death increases with increasing follow-up time, adjusted logistic regression models compared the odds of death between one and 15 days after discharge and 16 and 30 days after discharge.

A sensitivity analysis evaluated the odds of death between 11 and 30 days after discharge for patients alive at day 10 after admission with a hospital stay of 10 days or fewer, while controlling for other covariates in the primary multivariate model. We calculated odds ratios for the interaction of a one day incremental increase in length of stay over the study period and separately for each individual year of the study. Analyses were performed by Stata statistical software (version 14.0 StataCorp).

\section{Patient involvement}

No patients were involved in setting the research question or the outcome measures, nor were they involved in the design and implementation of the study. There are no plans to involve patients in the dissemination of results as the nature of the data precludes individual patient identification.

\section{Results}

A total of 188208 patients admitted with a hip fracture between 2000 and 2011 met inclusion criteria; 169258 were treated surgically and 18950 treated non-surgically. The average length of hospital stay was 8.1 days (range 1-995 days; standard deviation 8.3). During their hospital stay, 7364 (3.9\%) patients died, leaving 180844 survivors for post-discharge analysis. During the 30 days after discharge, another 9179 patients died (5.1\% mortality). During the study, the average length of stay decreased from 12.9 days in 2000 to 5.6 days in 2011 .

Most patients were admitted to hospital for fewer than 10 days (patient characteristics shown in table $2 \Downarrow$ ). For surgically treated patients, the average time to surgery was 1.8 days (standard deviation 2.1, range $0-128$ ); a longer time to surgery was associated with longer hospital stay. The percentage of patients with most comorbid conditions increased with increasing length of stay.

On univariate analysis, multiple factors were associated with increased 30 day mortality including non-surgical treatment, male sex, being white, older age, longer time to surgery, blood transfusion, comorbid conditions, and discharge to hospice (table $3 \Downarrow$ ). A shorter hospital stay ( $<5$ days and $<10$ days) was also associated with decreased 30 day mortality. Mortality rates did not change between the first six years and the last six years of the study. The mortality rate for surgically treated patients 
with hip fracture was $4.5 \%$ (7407/164 251) in the 30 days after discharge. The corresponding rate for non-surgically treated patients with hip fracture was $10.7 \%$ (1772/16 593).

On multivariate regression analysis, discharge to a hospice facility and age older than 90 years were the largest risk factors for 30 day mortality. A hospital stay longer than 10 days, compared with a stay of five days or fewer, was associated with increased odds of mortality during the first 30 days after discharge.

On Kaplan-Meier analysis, patients with a longer hospital stay had a lower rate of 30 day survival after discharge (fig $1 \Downarrow$ ).

Length of stay was not associated with mortality rates for non-surgically treated patients, when analyzed separately (fig 1). Patients in New York state with a hospital stay longer than 14 days and patients managed non-surgically had similar rates of 30 day survival after hospital discharge. For all patients with hip fracture and for those receiving surgical treatment only, increased length of stay was associated with increased early mortality in both groups.

\section{Sensitivity analyses}

The sensitivity analysis attempted to replicate the sensitivity analysis performed in a paper that evaluated mortality against length of hospital stay after hip fracture in the Swedish population. ${ }^{3}$ For patients alive at day 10 after admission with a length of hospital stay of 10 days or less, each one day increase in stay was associated with an $8 \%$ increased odds of death during 11-30 days from hospital admission overall (95\% confidence interval 1.07 to $1.10 ; \mathrm{P}<0.001$ for a one day increase in length of stay). The interaction variable (each one day incremental increase in length of stay over one day) was significantly associated with increased odds of mortality in seven of the 12 years in the study (web table 1).

\section{Subgroup analysis}

After adjustment for comorbid characteristics, injury, and demographics, each year in 2000-11 demonstrated a consistent trend of increasing odds of mortality 30 days after hospital discharge with increased length of hospital stay (fig $2 \Downarrow$ ). Web table 2 shows a similar trend in unadjusted odds ratios for 30 day mortality. Year 2009 represented an outlier when the association between hospital length of stay and 30 day mortality was especially pronounced. Exclusion of patients sustaining a fracture in 2009 from the primary adjusted analysis did not substantially alter the findings in this study.

For all discharge dispositions other than patients who went to hospices or left hospital against medical advice, we saw a significant association between hospital stays longer than 14 days and increased odds of 30 day mortality (web table 3 ). In the skilled nursing facility and inpatient rehabilitation subgroups (the most common dispositions), longer stay was associated with significantly higher mortality odds at stays of 6-10 days, 11-14 days, and longer than 14 days in the adjusted model, when compared with a stay of 1-5 days. For patients discharged to return home, hospital stays longer than 14 days was significantly associated with increased 30 day mortality, but no significant association was seen for shorter stays. Hospital stays longer than five days was associated with significantly higher mortality odds in patients with cancer. For patients with dementia, congestive heart failure, and chronic obstructive pulmonary disease, we saw significantly increased mortality odds for stays longer than 10 days but not for stays lasting six to 10 days. In the subgroup analysis for comorbid conditions, no association was seen between longer hospital stay and decreased odds of
30 day mortality after discharge (web table 4). Hospital stays longer than 10 days were associated with similarly elevated odds of mortality during days 1-15 and 16-30 after discharge.

\section{Discussion}

In this study of patients from New York state with hip fracture, a shorter inpatient hospital stay was associated with increased odds of survival at 30 days after discharge. The link between length of stay and mortality is important and likely to be multifactorial. Previous studies and our results suggest that an increased comorbidity burden is associated with increased length of hospital stay, ${ }^{9}{ }^{10}$ which highlights the relation between a patient's health status before injury and hospital length of stay.

\section{Clinical implications of results}

These findings contrast sharply with the results from a recent Swedish study by Nordström and colleagues, in which shortened hospital stay was associated with increased rates of early mortality. ${ }^{3}$ These conflicting results suggest that different associations with mortality rates exist in these two distinct populations. The Swedish cohort contained 116111 patients who were managed surgically. In New York state, the prevalent standard of care for hip fracture is surgical treatment in all patients who can tolerate surgery. Non-surgical management is chosen for those patients deemed medically unfit for surgery, unwilling to consent for surgery, or with a short life expectancy where palliative measures are deemed more appropriate.

In our study, non-surgically managed patients fared predictably poorly regardless of length of hospital stay. However, exclusion of these patients from the fracture cohort did not alter the overall improved mortality rates in patients with shorter hospital stays. The Nordström study did not assess the number of patients managed non-surgically or their outcomes with regards to mortality as a function of length of hospital stay. The lack of a non-surgical group likely represents different standards of care for hip fracture in Sweden, where virtually all patients with hip fracture are treated surgically.

Differences also exist in time to management of hip fracture; standard of care in Sweden involves fracture stabilization within 24 h. In our New York state cohort, 19.4\% (31 987/164 251) of patients waited for more than two days for surgery, and increased time to surgery was associated with longer hospital stay. With the generally accepted association in the hip fracture literature between longer time to surgery and increased mortality, ${ }^{11}{ }^{12}$ the extended time to surgery in our cohort would predict higher mortality. However, despite fewer patients treated surgically and longer mean time to surgery, mortality at 30 days after discharge in our cohort was lower than the Swedish study's cohort $(5.1 \% v 5.8 \%)$. Inpatient mortality was also lower in our cohort (3.9\% v 5.0\%), although patient factors could differ among populations. Our findings are in line with those of Grimes and colleagues, who found that time to surgery longer than 24 $\mathrm{h}$ was not associated significantly with 30 day mortality after adjusting for comorbid conditions. ${ }^{13}$ There is no way to know whether the mean 0.8 day difference in time to surgery between the Swedish and New York state cohorts (mean $1 v 1.8$ days) contributes significantly to mortality. However, it may be that early surgery is more important than surgery within $24 \mathrm{~h}$, as seen by the respective differences in mortality.

The effects of discharge destination on short term risk of death after hip fracture in Sweden has also been evaluated. ${ }^{14}$ In a recent Swedish study of 89301 patients from 2004 to 2012, most patients were discharged to nursing homes $(61.8 \%)$ or their own homes $(31.1 \%)$. After adjustment for comorbid characteristics 
and functional level, discharge to a nursing home (and especially a short term nursing home) after a hospital stay of up to 10 days was associated with an increased risk of early mortality. By contrast, only $12.9 \%(n=23272)$ of patients in our New York state cohort were discharged to their own homes; the vast majority of patients were discharged to short or long term care facilities where longer hospital stay was associated with higher odds of mortality at 30 days after discharge.

Finally, notable differences exist in length of hospital stay between the Swedish and New York state cohorts. In the New York state cohort, where insurers exert pressure to discharge patients to less costly rehabilitation facilities, nearly $40 \%$ of patients were discharged within five days and $82 \%$ discharged within 10 or fewer days. By contrast, only $18 \%$ of patients were discharged within five days and less than $50 \%$ were discharged in 10 days or less in Sweden, where a publicly financed system might not have the same incentives. These major differences in financial considerations and mean length of stay after hip fracture could partly explain the contradictory results seen in the two studies.

Other authors have attempted to determine the relation between length of hospital stay and mortality or other outcomes in patients with hip fracture. Kondo and colleagues compared length of stay and mortality after hip fractures between three Japanese and two US hospitals with vastly different lengths of stay. ${ }^{15}$ Although the researchers found lower mortality in the Japanese hospitals with longer lengths of stay, the small sample size make interpretation of the results challenging.

Dubljanin-Respovic and colleagues found no effect of length of stay on one year mortality in a consecutive series of 228

Serbian patients with hip fracture, ${ }^{16}$ and Heyes and colleagues found increased readmission rate was associated with increasing length of stay after hip fracture in a Northern Ireland hospital system. $^{17}$

The association between length of hospital stay and mortality has also been studied in populations with non-hip fracture. Kaboli and colleagues analyzed over four million admissions through the US Veterans Affairs Medical System and found length of hospital stay decreasing for all diagnoses by 1.46 days and a concomitant decrease in all cause mortality at 90 days after discharge. ${ }^{18}$ In patients with acute heart failure, Reynolds and colleagues found an association between longer initial hospital stay and increased all cause mortality. ${ }^{19}$ Both of these studies included a US population and support the findings of an association between shorter hospital stay and decreased mortality in New York state patients with hip fracture. The contradictory results between US studies and those found in data from the Swedish hip fracture registry highlight the challenges in interpreting studies on length of hospital stay across populations with different systems of care.

\section{Strengths and limitations}

Our study cohort could vary from other statewide populations in the USA, and thus our findings might not be generalizable to populations in other US states or countries. Administrative claims data could be incomplete or contain inaccurate coding of diagnoses and comorbid conditions, and different administrative databases could contain different rates of comorbid conditions. ${ }^{20}$ However, comorbidities that would affect reimbursement and acuity indices would likely have been included. The SPARCS dataset does not contain laboratory values or permit assessment of the severity of comorbid illness, and it does not include the American Society of Anesthesiologists classification.
The fracture date was not recorded in relation to admission date. Some patients sustain a hip fracture while in hospital for another condition, biasing these patient's records toward a longer stay. To control for this effect, we purposely included only patients whose admission or primary diagnosis was for hip fracture.

Patient socioeconomic status could affect access to care in New York state more than in single nation European populations, ${ }^{4}$ but is not included in the SPARCS database and therefore could not be assessed as a covariate. The original study design planned to use Cox regression models but the assumption of proportional hazards was not met. Use of a logistic regression model represents a deviation from the original study design and may be considered a limitation. Finally, specific cause of death was not available because few autopsies are performed in this population. Strengths of this study included its similarity to the Swedish dataset in terms of patient numbers, use of unique patient identifiers to allow linkage to statewide death registries, and high completeness in capturing a single population with a different system of care.

\section{Conclusion}

The relation between length of hospital stay and 30 day post-discharge mortality after hip fracture in New York state differs significantly from that seen in a Swedish population.

Earlier hospital discharge in New York state is associated with improved survival, and longer length of hospital stay probably represents a surrogate for medical comorbidities or complications occurring in the hospital that delayed a safe, early discharge. This critical difference suggests prolonging admission to hospital would not improve mortality outcomes in a New York state population. Caution should be used in extrapolating results of population based studies when healthcare systems are dissimilar.

Contributors: LEN and SLK contributed equally to this work; LEN, SLK, MS, and JE contributed to the conception or design of the work; LEN, MM, BM, and JCE contributed to the acquisition of data; LEN, SLK, MM, BM, and JCE contributed to the analysis; LEN, SLK, MS, MM, BM, and JCE contributed to the interpretation of data for the work; LEN, SK, MS, MM, BM, and JCE contributed to drafting the work and revising it critically for important intellectual content; LEN, SLK, MS, MM, BM, and JCE gave final approval of the version to be published; LEN, SLK, MS, MM, BM, and JCE agree to be accountable for all aspects of the work in ensuring that questions related to the accuracy or integrity of any part of the work are appropriately investigated and resolved. JCE and LEN are the guarantors.

Funding: This study was supported by the University of Rochester Clinical Translational Science Institute award number UL1 TR000042 from the National Center for Advancing Translational Sciences of the National Institutes of Health and grant K-08 AR060164-01A from the National Institutes of Health. Funding was used for expenses related to biostatistics consultation. The funding bodies had no role in study design, analysis, or reporting.

Competing interests: All authors have completed the ICMJE uniform disclosure form at www.icmje.org/coi_disclosure.pdf and declare: support from the National Institutes of Health for the submitted work; no financial relationships with any organizations that might have an interest in the submitted work in the previous three years; no other relationships or activities that could appear to have influenced the submitted work.

Ethical approval: This study was approved by the New York State Department of Health, Bureau of Health informatics data protection review board, New York State Department of Vital Statistics, New York City Department of Health and Mental Hygiene, and University of Rochester research subjects review board. 


\section{What is already known on this topic}

Shorter hospital stays have been associated with increased odds of mortality in a Swedish population

In New York state, increased comorbid conditions are associated with increased costs and longer hospital stays following hip fracture

\section{What this paper adds}

Hospital stays longer than 10 days were associated with increased odds of death at 30 days after hospital discharge in patients in New York state

Non-surgical treatment of hip fractures was associated $10.7 \%$ mortality compared with $4.5 \%$ treated surgically at 30 days after discharge Risk factors associated with early mortality include discharge to a hospice facility, older age, metastatic disease, and non-surgical management

Data sharing: No additional data are available from the authors owing to disclosure agreements with New York Statewide Planning and Research Cooperative System (SPARCS). Data may be obtained through SPARCS at https://www.health.ny.gov/statistics/sparcs/access/

LEN and JCE affirm that the manuscript is an honest, accurate, and transparent account of the study being reported; no important aspects of the study have been omitted; and any discrepancies from the study as planned have been explained.

1 Brauer CA, Coca-Perraillon M, Cutler DM, et al. Incidence and mortality of hip fractures in the United States. JAMA 2009;302:1573-9.

2 Fenton JJ, Jerant AF, Bertakis KD, et al. The cost of satisfaction: a national study of patient satisfaction, health care utilization, expenditures, and mortality. Arch Intern Med 2012;172:405-11.

3 Nordstrom P, Gustafson Y, Michaelsson K, et al. Length of hospital stay after hip fracture and short term risk of death after discharge: a total cohort study in Sweden. BMJ 2015;350:h696.

4 Goodman DC, Fisher ES, Chang CH. After hospitalization: a Dartmouth Atlas report on post-acute care for Medicare beneficiaries. A report of the Dartmouth Atlas project. 2011 www.dartmouthatlas.org/downloads/reports/Post_discharge_events_092811.pdf.

5 Lahtinen A, Leppilahti J, Harmainen S, et al. Geriatric and physically oriented rehabilitation improves the ability of independent living and physical rehabilitation reduces mortality: a randomised comparison of 538 patients. Clin Rehabil 2015;29:892-906.

6 Royal College of Physicians. National Hip Fracture Database. National report 2012. www. nhfd.co.uk.

7 Boockvar KS, Halm EA, Litke A, et al. Hospital readmissions after hospital discharge for hip fracture: surgical and nonsurgical causes and effect on outcomes. J Am Geriatr Soc 2003:51:399-403.

8 Stagg V. CHARLSON: Stata module to calculate Charlson index of comorbidity. 2006. http://econpapers.repec.org/software/bocbocode/s456719.htm.

9 Nikkel LE, Fox EJ, Black KP, et al. Impact of comorbidities on hospitalization costs following hip fracture. J Bone Joint Surg Am 2012;94:9-17.

10 Roche JJ, Wenn RT, Sahota O, et al. Effect of comorbidities and postoperative complications on mortality after hip fracture in elderly people: prospective observational cohort study. BMJ 2005;331:1374
11 Bottle A, Aylin P. Mortality associated with delay in operation after hip fracture: observational study. BMJ 2006;332:947-51

12 Moran CG, Wenn RT, Sikand M, et al. Early mortality after hip fracture: is delay before surgery important? J Bone Joint Surg Am 2005;87:483-9.

13 Grimes JP, Gregory PM, Noveck H, et al. The effects of time-to-surgery on mortality and morbidity in patients following hip fracture. Am J Med 2002;112:702-9.

14 Nordstrom P, Michaelsson K, Hommel A, et al. Geriatric rehabilitation and discharge location after hip fracture in relation to the risks of death and readmission. J Am Med Dir Assoc 2015, doi:10.1016/j.jamda.2015.07.004

15 Kondo A, Zierler BK, Isokawa Y, et al. Comparison of lengths of hospital stay after surgery and mortality in elderly hip fracture patients between Japan and the United States-the relationship between the lengths of hospital stay after surgery and mortality. Disabil Rehabil 2010;32:826-35.

16 Dubljanin-Raspopovic E, Markovic-Denic L, Marinkovic $\mathrm{J}$, et al. Does early functional outcome predict 1 -year mortality in elderly patients with hip fracture? Clin Orthop 2013;471:2703-10

17 Heyes GJ, Tucker A, Marley D, et al. Predictors for readmission up to 1 year following hip fracture. Arch Trauma Res 2015;4:e27123.

18 Kaboli PJ, Go JT, Hockenberry J, et al. Associations between reduced hospital length of stay and 30-day readmission rate and mortality: 14-year experience in 129 Veterans Affairs hospitals. Ann Intern Med 2012;157:837-45.

19 Reynolds K, Butler MG, Kimes TM, et al. Relation of acute heart failure hospital length of stay to subsequent readmission and all-cause mortality. Am J Cardiol 2015;116:400-5.

20 Bohl DD, Russo GS, Basques BA, et al. Variations in data collection methods between national databases affect study results: a comparison of the nationwide inpatient sample and national surgical quality improvement program databases for lumbar spine fusion procedures. J Bone Joint Surg Am 2014;96:e193.

Accepted: 10 Nov 2015

\section{Cite this as: BMJ 2015;351:h6246}

(c) Nikkel et al 2015

This is an Open Access article distributed in accordance with the Creative Commons Attribution Non Commercial (CC BY-NC 4.0) license, which permits others to distribute, remix, adapt, build upon this work non-commercially, and license their derivative works on different terms, provided the original work is properly cited and the use is non-commercial. See: http://creativecommons.org/licenses/by-nc/4.0/. 


\section{Tables}

Table 1/ Coding algorithm for comorbid conditions in patients with hip fracture in New York State

\begin{tabular}{ll} 
Comorbidities & ICD-9-CM codes \\
Myocardial infarction & $410 . x, 412 . x$ \\
\hline Congestive heart failure & $398.91,402.01,402.11,402.91,404.01,404.03,404.11,404.13,404.91,404.93,425.4-425.9,428 . x$ \\
\hline Peripheral vascular disease & $093.0,437.3,440 . x, 441 . x, 443.1-443.9,47.1,557.1,557.9, \mathrm{~V} 43.4$ \\
\hline Cerebrovascular disease & $362.34,430 . x-438 . x$ \\
\hline Dementia & $290 . x, 331 . x, 437.0 x, 333.4,292.82,294.10,291.2 x, 292.82,24.10,294.11,294.20,294.21$ \\
\hline Chronic pulmonary disease & $416.8,416.9,490 . x-505 . x, 506.4,508.1,508.8$ \\
\hline Diabetes mellitus & $250.0-250.3,250.8,250.9$ \\
\hline Renal disease & $403.01,403.11,403.91,404.02,404.03,404.12,404.13,404.92,404.93,582 . x, 583.0-583.7,585 . x, 586 . x, 588.0$, V42.0, V45.1, \\
\hline Cancer & V56.x \\
\hline Metastatic disease & $140 . x-172 . x, 174 . x-195.8,200 . x-208 . x, 238.6, \mathrm{~V} 10 . x$ \\
\hline Diabetes with complications & $250.4-250.7$ \\
\hline
\end{tabular}




\begin{tabular}{|c|c|c|c|c|}
\hline \multicolumn{5}{|l|}{$\begin{array}{l}\text { Table 2| Characteristics of } 180844 \text { patients wit } \\
\text { patients who died before hospital discharge) }\end{array}$} \\
\hline & $0-5$ & 6-10 & $11-14$ & $>14$ \\
\hline \multicolumn{5}{|l|}{ Demographics and mortality } \\
\hline No of patients & $71780(39.7)$ & $76700(42.4)$ & $17063(9.4)$ & $15301(8.5)$ \\
\hline Female sex & $55119(76.8)$ & $57389(74.8)$ & $11964(70.1)$ & $9944(65.0)$ \\
\hline Mean age in years (mean (SD)) & $80.7(10.3)$ & $81.8(9.5)$ & $81.7(9.6)$ & $80.9(10.0)$ \\
\hline Non-surgical treatment & $8609(12.0)$ & $3987(5.2)$ & $1725(10.1)$ & $2272(14.8)$ \\
\hline 30 day mortality if treated surgically & $1864(3.0)$ & $2991(4.1)$ & $1033(6.7)$ & $1519(11.7)$ \\
\hline 30 day mortality if treated non-surgically & $910(10.6)$ & $446(11.2)$ & $171(9.9)$ & $245(10.8)$ \\
\hline \multicolumn{5}{|l|}{ Main diagnosis } \\
\hline Femoral neck fracture & $21184(29.5)$ & $22023(28.7)$ & $4677(27.4)$ & $4090(26.7)$ \\
\hline Intertrochanteric fracture & $50596(70.5)$ & $54677(62.3)$ & $12386(72.6)$ & $11211(73.3)$ \\
\hline \multicolumn{5}{|l|}{ Surgical variables } \\
\hline Hemiarthroplasty & $17084(23.8)$ & $22756(29.7)$ & $5084(29.8)$ & $4303(28.1)$ \\
\hline Total hip arthroplasty & $1820(2.5)$ & $2224(2.9)$ & $442(2.6)$ & $371(2.4)$ \\
\hline Open reduction internal fixation & $29379(40.9)$ & $36083(47.0)$ & $7828(42.7)$ & $6620(43.3)$ \\
\hline Other fixation & $14933(20.8)$ & $11679(15.2)$ & $1994(11.7)$ & $1742(11.4)$ \\
\hline Time to surgery if treated surgically (mean (SD)) & $0.9(0.7)$ & $1.8(1.3)$ & $2.9(2.3)$ & $4.2(5.1)$ \\
\hline Required transfusion & $22551(31.4)$ & $33326(43.4)$ & $7996(46.9)$ & $7384(48.3)$ \\
\hline \multicolumn{5}{|l|}{ Comorbid conditions } \\
\hline Dementia & $16161(22.5)$ & $18120(23.6)$ & $4202(24.6)$ & $3698(24.2)$ \\
\hline Acute myocardial infarction & $3810(5.3)$ & $5597(7.3)$ & $1862(10.9)$ & $2033(13.3)$ \\
\hline Cerebrovascular disease & $3144(4.4)$ & $4531(5.9)$ & $1383(8.1)$ & $1350(8.8)$ \\
\hline Chronic obstructive pulmonary disease & $11640(16.2)$ & $14401(18.8)$ & $3736(21.9)$ & $3750(24.5)$ \\
\hline Diabetes mellitus & $11529(16.1)$ & $13942(18.2)$ & $3313(19.4)$ & $2817(18.4)$ \\
\hline Renal disease & $3603(5.0)$ & $4991(6.5)$ & $1463(8.6)$ & $1580(10.3)$ \\
\hline Cancer & $8819(12.3)$ & $9672(12.6)$ & $2097(12.3)$ & $1944(8.6)$ \\
\hline Metastatic disease & $660(0.9)$ & $940(1.2)$ & $270(1.6)$ & $377(2.5)$ \\
\hline Diabetes with complications & $884(1.2)$ & $1430(1.9)$ & $432(2.5)$ & $555(3.6)$ \\
\hline Congestive heart failure & $6726(9.4)$ & $11555(15.1)$ & $3835(22.5)$ & $4448(29.1)$ \\
\hline Weighted Charlson score (mean (SD)) & $0.9(1.2)$ & $1.1(1.3)$ & $1.4(1.4)$ & $1.6(1.6)$ \\
\hline \multicolumn{5}{|l|}{ Discharge disposition } \\
\hline Long term care & $519(0.7)$ & $746(1.0)$ & $145(0.8)$ & $191(1.2)$ \\
\hline Skilled nursing facility & $39691(55.3)$ & $47059(61.4)$ & $10485(61.4)$ & $9443(61.7)$ \\
\hline Left hospital against medical advice & $360(0.5)$ & $81(0.1)$ & $22(0.1)$ & $30(0.2)$ \\
\hline Cancer hospital & $6016(8.4)$ & $8013(10.4)$ & $1677(9.8)$ & $1197(7.8)$ \\
\hline Home & $4921(6.9)$ & $4823(6.3)$ & $1456(8.5)$ & $1511(9.9)$ \\
\hline Home health agency & $4116(5.7)$ & $3571(4.7)$ & $1101(6.5)$ & $1280(8.4)$ \\
\hline Hospice & $333(0.5)$ & $314(0.4)$ & $149(0.9)$ & $291(1.9)$ \\
\hline Inpatient hospital & $3063(4.3)$ & $1174(1.5)$ & $297(1.7)$ & $289(1.9)$ \\
\hline Inpatient rehabilitation & $12641(17.6)$ & $10791(14.1)$ & $1704(10.0)$ & $1024(6.7)$ \\
\hline
\end{tabular}




\section{Table 3| Variables associated with odds of $\mathbf{3 0}$ day mortality following hospital discharge for femoral neck fracture (excluding patients who died before discharge)}

\begin{tabular}{|c|c|c|c|c|c|}
\hline & \multicolumn{3}{|c|}{ Unadjusted analysis } & \multicolumn{2}{|c|}{ Adjusted analysis } \\
\hline & Survived $(n=171665)$ & Deceased $(n=9179)$ & $\mathbf{P}$ & Odds ratio $(95 \% \mathrm{Cl})$ & $\mathbf{P}$ \\
\hline No surgery & $14821(8.63)$ & $1772(19.3)$ & $<0.001$ & 1.95 (1.80 to 2.12$)$ & $<0.001$ \\
\hline Female sex & $128467(74.84)$ & $5949(64.81)$ & $<0.001$ & 0.59 (0.56 to 0.62$)$ & $<0.001$ \\
\hline \multicolumn{6}{|l|}{ Ethnic origin } \\
\hline White & $147800(86.1)$ & $8157(88.9)$ & $<0.001$ & Reference & - \\
\hline Black & $7188(4.2)$ & $278(3)$ & $<0.001$ & 0.70 (0.61 to 0.79$)$ & $<0.001$ \\
\hline Other & $13792(8)$ & $639(7)$ & $<0.001$ & 0.92 (0.84 to 1.01$)$ & 0.07 \\
\hline Age (mean (SD)) & $81.0(9.9)$ & $86.0(8.0)$ & $<0.001$ & - & - \\
\hline $50-59$ years & $7411(4.3)$ & $66(0.7)$ & $<0.001$ & Reference & - \\
\hline $60-69$ years & $14947(8.7)$ & $296(3.2)$ & $<0.001$ & 1.87 (1.42 to 2.48$)$ & $<0.001$ \\
\hline 70-79 years & $40107(23.4)$ & $1295(14.1)$ & $<0.001$ & 2.68 (2.07 to 3.47 ) & $<0.001$ \\
\hline $80-89$ years & $77166(45)$ & $4250(46.3)$ & 0.011 & 4.24 (3.28 to 5.48$)$ & $<0.001$ \\
\hline$>90$ years & $32034(18.7)$ & $3272(35.7)$ & $<0.001$ & 7.63 (5.90 to 9.86$)$ & $<0.001$ \\
\hline Femoral neck fracture & 49407 (28.78) & $2567(27.97)$ & 0.09 & $1.01(0.96$ to 1.06$)$ & 0.7 \\
\hline Days to surgery (mean (SD)) & $1.7(2.0)$ & $2.2(3.0)$ & $<0.001$ & - & - \\
\hline$>2$ days & $29903(19.1)$ & $1994(26.9)$ & $<0.001$ & $1.11(1.05$ to 1.18$)$ & $<0.001$ \\
\hline Received transfusion & $67119(39.1)$ & $4138(45.1)$ & $<0.001$ & 1.19 (1.14 to 1.25$)$ & $<0.001$ \\
\hline \multicolumn{6}{|l|}{ Comorbidities } \\
\hline Dementia & $38338(22.3)$ & $3843(41.9)$ & $<0.001$ & 2.02 (1.92 to 2.12$)$ & $<0.001$ \\
\hline Cardiac disease (acute) & $12192(7.1)$ & $1110(12.1)$ & $<0.001$ & $1.23(1.15$ to 1.33$)$ & $<0.001$ \\
\hline Cerebrovascular disease & $9700(5.7)$ & $708(7.7)$ & $<0.001$ & 1.20 (1.10 to 1.31$)$ & $<0.001$ \\
\hline Chronic obstructive pulmonary disease & $31199(18.2)$ & $2328(25.4)$ & $<0.001$ & 1.44 (1.37 to 1.52$)$ & $<0.001$ \\
\hline Diabetes & $30057(17.5)$ & $1544(16.8)$ & 0.09 & $1.01(0.95$ to 1.08$)$ & 0.7 \\
\hline Renal disease & $10583(6.2)$ & $1054(11.5)$ & $<0.001$ & $1.43(1.33$ to 1.55$)$ & $<0.001$ \\
\hline Cancer & $20969(12.2)$ & $1563(17.0)$ & $<0.001$ & $1.18(1.10$ to 1.26$)$ & $<0.001$ \\
\hline Metastatic disease & $1840(1.1)$ & $407(4.4)$ & $<0.001$ & 3.54 (3.08 to 4.07 ) & $<0.001$ \\
\hline Diabetes with complications & $3141(1.8)$ & $160(1.7)$ & 0.55 & 1.02 (0.86 to 1.22$)$ & 0.8 \\
\hline Congestive heart failure & $23796(13.9)$ & $2768(30.2)$ & $<0.001$ & $1.76(1.67$ to 1.85$)$ & $<0.001$ \\
\hline Weighted Charlson sum (mean (SD)) & $1.0(1.3)$ & $1.8(1.8)$ & $<0.001$ & - & - \\
\hline \multicolumn{6}{|l|}{ Length of stay (days) } \\
\hline $1-5$ & $69006(40.2)$ & $2774(30.2)$ & $<0.001$ & Reference & - \\
\hline $6-10$ & $73263(42.7)$ & $3437(37.4)$ & $<0.001$ & $1.06(0.98$ to 1.15$)$ & 0.1 \\
\hline $11-14$ & $15859(9.2)$ & $1204(13.1)$ & $<0.001$ & $1.32(1.19$ to 1.47$)$ & $<0.001$ \\
\hline$>14$ & $13537(7.9)$ & $1764(19.2)$ & $<0.001$ & 2.03 (1.84 to 2.24$)$ & $<0.001$ \\
\hline \multicolumn{6}{|l|}{ Discharge disposition } \\
\hline Skilled nursing facility & $100454(58.5)$ & $6224(67.8)$ & $<0.001$ & Reference & - \\
\hline Long term care & $1507(0.9)$ & $94(1)$ & 0.145 & $1.10(0.88$ to 1.36$)$ & 0.4 \\
\hline Left hospital against medical advice & $474(0.3)$ & $19(0.2)$ & 0.216 & $0.7(0.44$ to 1.12$)$ & 0.1 \\
\hline Cancer center & $16400(9.6)$ & $503(5.5)$ & $<0.001$ & $0.69(0.62$ to 0.76$)$ & $<0.001$ \\
\hline Home & $12317(7.2)$ & $394(4.3)$ & $<0.001$ & $0.68(0.61$ to 0.76$)$ & $<0.001$ \\
\hline Home health & $9825(5.7)$ & $243(2.7)$ & $<0.001$ & 0.50 (0.43 to 0.57$)$ & $<0.001$ \\
\hline Hospice & $224(0.1)$ & $863(9.4)$ & $<0.001$ & 37.9 (32.34 to 44.42$)$ & $<0.001$ \\
\hline Inpatient hospital & $4488(2.6)$ & $335(3.7)$ & $<0.001$ & $1.20(1.06$ to 1.36$)$ & 0.005 \\
\hline Inpatient rehabilitation & $25678(15)$ & $482(5.3)$ & $<0.001$ & 0.47 (0.43 to 0.52$)$ & $<0.001$ \\
\hline Year 2006-11 & $85553(49.8)$ & $4505(49.1)$ & 0.157 & $0.74(0.68$ to 0.80$)$ & $<0.001$ \\
\hline Length of stay 6-10 days during years $2006-11$ & - & - & - & $1.08(0.97$ to 1.20$)$ & 0.1692 \\
\hline Length of stay $11-14$ days during years $2006-11$ & - & - & - & $1.26(1.09$ to 1.47$)$ & 0.002 \\
\hline Length of stay $>14$ days during years $2006-11$ & - & - & - & 1.25 (1.09 to 1.43 ) & 0.001 \\
\hline
\end{tabular}


Table 3 (continued)

Unadjusted analysis

Survived $(n=171665)$ Deceased $(n=9179)$
Adjusted analysis

Odds ratio $(95 \% \mathrm{Cl})$ 


\section{Figures}

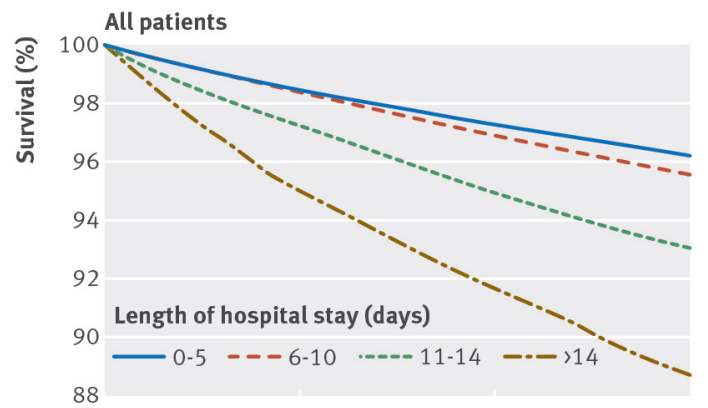

No at risk

0-5 days

$\begin{array}{lll}71780 & 70686 & 69803\end{array}$

6-10 days

$\begin{array}{llll}76700 & 75530 & 74376 & 73357\end{array}$

11-14 days

$\begin{array}{llll}17063 & 16605 & 16217 & 15881\end{array}$

$>14$ days

$\begin{array}{llll}15301 & 14558 & 14044 & 13579\end{array}$

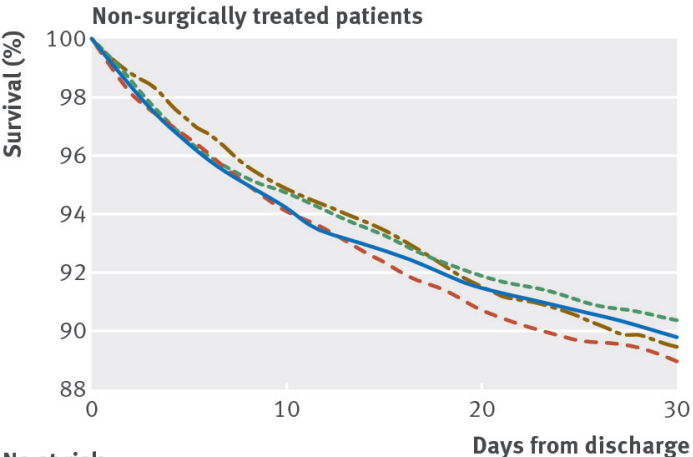

No at risk

Days from discharge

0-5 days

$\begin{array}{rccc}8609 & 8112 & 7861 & 7713 \\ \begin{array}{r}\text { 6-10 days } \\ 3987\end{array} & 3762 & 3636 & 3553 \\ \begin{array}{r}11-14 \text { days } \\ 1725\end{array} & 1633 & 1584 & 1556 \\ \begin{array}{r}14 \text { days } \\ 2272\end{array} & 2156 & 2081 & 2031\end{array}$

Fig 1 Kaplan-Meier graphs showing survival at 30 days after hospital discharge for patients with hip fracture. Graphs are separated by groups of all patients $(n=180844)$ and of non-surgically treated patients $(n=16593)$

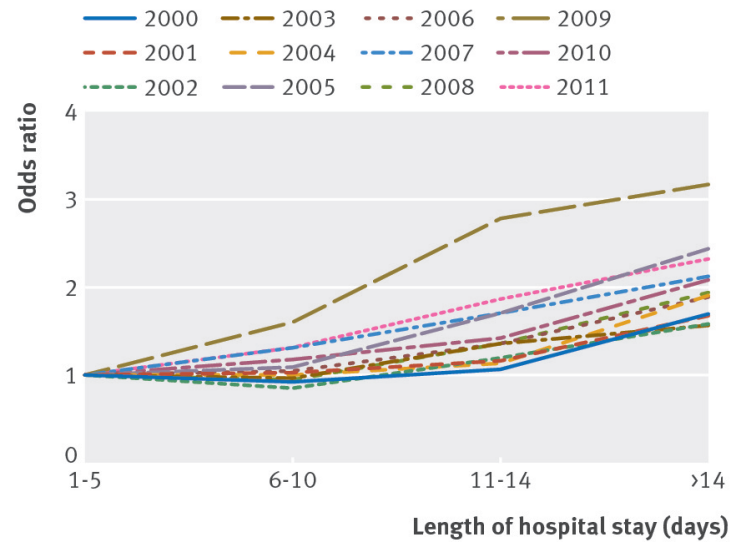

Fig 2 Adjusted odds ratios for mortality at 30 days after hospital discharge for patients with hip fracture, with adjustment for comorbid characteristics, injury, and demographics at each year in 2000-11 